\title{
The impact of 3D fields on tearing mode stability of H-modes
}

\author{
R.J. Buttery ${ }^{1}$, S. Gerhardt ${ }^{2}$, R.J. La Haye ${ }^{1}$, Y.Q. Liu $^{3}$, \\ H. Reimerdes ${ }^{4}$, S. Sabbagh ${ }^{4}$, M.S. Chu ${ }^{1}$, T.H. Osborne ${ }^{1}$, \\ J.-K. Park ${ }^{2}$, R.I. Pinsker ${ }^{1}$, E.J. Strait ${ }^{1}$, J.H. Yu ${ }^{5}$, and the DIII-D \\ and NSTX Teams \\ ${ }^{1}$ General Atomics, PO Box 85608, San Diego, CA 92186-5608, USA \\ ${ }^{2}$ Princeton Plasma Physics Laboratory, PO Box 451, Princeton, NJ 08543-0451, USA \\ ${ }^{3}$ EURATOM/CCFE Fusion Association, Culham Science Centre, Abingdon OX14 3DB, UK \\ ${ }^{4}$ Department of Applied Physics and Applied Mathematics, Columbia University, 2960 \\ Broadway, New York, NY 10027-6900, USA \\ ${ }^{5}$ Center for Energy Research, University of California-San Diego, 9500 Gilman Dr, La Jolla, \\ CA 92093-0417, USA
}

E-mail: buttery@ fusion.gat.com

Received 14 January 2011, accepted for publication 5 May 2011

Published 31 May 2011

Online at stacks.iop.org/NF/51/073016

\begin{abstract}
New processes have been discovered in the interaction of 3D fields with tearing mode stability at low torque and modest $\beta$ on DIII-D and NSTX. These are thought to arise from the plasma response at the tearing resonant surface, which theoretically is expected to depend strongly on plasma rotation and underlying intrinsic tearing stability. This leads to sensitivities additional to those previously identified at low density where the plasma rotation is more readily stopped, or at high $\beta_{\mathrm{N}}$ where ideal MHD responses amplify the fields (where $\beta_{\mathrm{N}}$ is the plasma $\beta$ divided by the ratio of plasma current to minor radius multiplied by toroidal field). It is found that the threshold size for 3D fields to induce modes tends to zero as the natural tearing $\beta_{\mathrm{N}}$ limit is approached. 3D field sensitivity is further enhanced at low rotation, with magnetic probing detecting an increased response to applied fields in such regimes. Modelling with the MARS-F code confirms the interpretation with the usual plasma screening response breaking down in low rotation plasmas and a tearing response developing, opening the door to additional sensitivities to $\beta$ and the current profile. Typical field thresholds to induce modes in torque-free $\beta_{\mathrm{N}} \sim 1.5 \mathrm{H}$-modes are well below those in ohmic plasmas or plasmas near the ideal $\beta_{\mathrm{N}}$ limit. The strong interaction with the tearing mode $\beta_{\mathrm{N}}$ limit is identified through rotation shear, which is decreased by the 3D field, leading to decreased tearing stability. Thus both locked and rotating mode field thresholds can be considered in terms of a torque balance, with sufficient braking leading to destabilization of a mode. On this basis new measurements of the principal parameter scalings for error field threshold have been obtained in torque-free H-modes leading to new predictions for error field sensitivity in ITER. The scalings have similar exponents to ohmic plasmas, but with seven times lower threshold at the ITER baseline $\beta_{\mathrm{N}}$ value of 1.8 , and a linear dependence on proximity to the tearing mode $\beta_{\mathrm{N}}$ limit ( 2.2 at zero torque). This reinforces the need to optimize error field correction strategies in ITER, and implement sources to drive plasma rotation.
\end{abstract}

(Some figures in this article are in colour only in the electronic version)

\section{Introduction}

The effects of low mode number 3D fields have long been a cause of concern for tokamaks. Such fields, commonly termed 'error fields', naturally arise from asymmetries in device design and construction. Harmonics of the fields can resonate with rational surfaces in the plasma, potentially driving confinementdegrading magnetic islands. However, when the plasma is rotating, the fields are generally shielded out by image currents at the rational surface. But with finite resistivity this interaction generates a torque [1] that reduces the rotation and changes the phase of the imaging response from perfect shielding, enabling slight tearing. If the field increases, this torque and consequent phase shift get larger, leading to less perfect shielding and further increased torque until a bifurcation point is reached, termed 'penetration' - a transition to large scale tearing and braking, usually leading to a stationary 'locked' mode and plasma termination [2]. The most performancelimiting of such modes is generally the $m / n=2 / 1$ tearing mode (denoting poloidal/toroidal mode number). 
This process was initially thought to pose the greatest risk to low density ohmic operation, where the rational $q=2$ surface can be readily stopped due to its low inertia, low viscosity and weak coupling to the bulk plasma; error field correction systems were designed for ITER on this basis [3]. More recently the ideal shielding response has been generalized to the whole plasma [4], and is then found to drive a kink-like response, as well as shielding on other resonant surfaces. The resulting perturbed currents amplify the applied fields across the plasma, leading to increased sensitivity as the ideal kink $\beta$ limit is approached [5] and to changes in the structure of the perturbed field at the resonant surface at all $\beta$ [6]. The consequences of this on tolerable error field levels and structures in high $\beta$ high confinement $\mathrm{H}$-modes were set out in [7], where a reduction in error field tolerance with $\beta$ [8] was linked to increases in plasma response, particularly as $\beta$ rose above the no-wall ideal-MHD limit. This highlighted the potential for error fields to trigger tearing modes in H-modes, particularly at high $\beta$ or as torque is lowered.

However, from the underlying theory [1] it is clear that further effects associated with the plasma response directly at the resonant surface can play a role. Firstly, if plasma rotation is lowered, then the shielding response will decrease, leading to increased residual tearing and a stronger coupling to the external field. Secondly, if underlying tearing stability is weaker, the partially shielding-suppressed island will be driven to larger amplitude by the error field, again increasing coupling to the external field and likelihood of mode penetration. These effects will only occur when the shielding response is susceptible to weakening-either at low rotation or with weak tearing stability. This turns out to be precisely the regime where we expect future burning devices to operate, which will have low rotation due to their size and low injected torque. Recent studies $[9,10]$ also show that lower rotation regimes have weaker intrinsic tearing stability, with $2 / 1$ modes growing out of the noise at lower $\beta_{\mathrm{N}}$ values. This linkage also raises the prospect of a third mechanism of mode triggeringif electromagnetic braking slows rotation sufficiently it may make the plasma intrinsically tearing unstable. Evidence of all three of these effects is presented in this paper.

Experiments were executed on DIII-D and NSTX to explore these potential resonant surface effects. In particular, the expected increasing sensitivity to error fields in plasmas with decreased tearing stability or decreased rotation is confirmed on DIII-D (section 2) using $n=1$ fields applied with the 12 segment 'I-coil' set. The conceptual explanation of these effects through a decrease in shielding is confirmed by modelling with the MARS-F code (section 3). The way in which the fields act on plasma rotation to change tearing stability is elucidated further in parallel experiments on NSTX (section 4), where an action through rotation braking is determined. Finally, scalings for error field thresholds to trigger $2 / 1$ modes have been obtained for $\mathrm{H}$-modes with no injected torque on DIII-D (section 5), indicating thresholds seven times lower than previous ohmic scalings. The conclusion and implications of this work are discussed in section 6. We start, however, with a discussion of how to calculate the applied fields and measure correction requirements.
1.1. Calculating the applied field and accounting for the harmonic mix in DIII-D

To predict requirements for future devices, 3D field thresholds must be couched in terms of tolerable external fields. However, from the above arguments, the total resonant field at a given surface of interest depends strongly on the plasma ideal response. Calculating this with the IPEC code [7,11] for DIII-D plasmas similar to those used in the experiments described here (section 2) indicates a total resonant field at $q=2$ of $3.26 \mathrm{G} \mathrm{kA}^{-1}$ of I-coil current, compared with $1.1 \mathrm{GkA}^{-1}$ of vacuum field. The vacuum field is not an appropriate measure of external field, as other external field harmonics couple through the plasma to make a $2 / 1$ field at $q=2$. However, other measures of total field amplitude are similarly problematic, as the plasma response will depend on the harmonic content of the external fields. A solution emerges from IPEC, which identifies a single 'dominant' eigenstructure of field at the plasma boundary that generates resonant fields at $q=2[11]$ - it is the degree to which this component is applied which governs the amount of resonant $q=2$ field. This can be computed by an overlap integral of the applied boundary field with this 'dominant' eigenstructure, providing a consistent basis to quantify applied fields of different structure and modetrigger thresholds. For the DIII-D I-coils and this particular plasma configuration, this 'dominant eigenmode' approach yields a 'resonant boundary field' strength of $1.57 \mathrm{G} \mathrm{kA}^{-1}$, and this measure is used for the rest of this study. However, it should be noted that there may be further less 'dominant' field structures that contribute to braking and mode formationthis remains an area of ongoing research that may refine error correction requirements, and the need for one or more coil sets.

A further problem, when measuring applied fields, such as from the DIII-D I-coils discussed in section 2, is that it is also important to account for intrinsic error field sources, which may differ in harmonic content and resonances. This can be addressed by representing the applied (I-coil) field and intrinsic error field by equivalent distributions of normal magnetic field on an external reference surface such as the plasma boundary. The intrinsic error field distribution, $B_{\mathrm{E}}$, can then be divided into two parts:

$$
B_{\mathrm{E}}=B_{\mathrm{EN}}+B_{\mathrm{EA}} \text {. }
$$

The 'non-aligned' part, $B_{\mathrm{EN}}$, is defined to have zero overlap integral with both the I-coil field, $B_{\mathrm{I}}$, and also with the 'aligned' part, $B_{\mathrm{EA}}$, to which the I-coil field adds linearly. The electromagnetic torque, $T$, from these fields is proportional to $\left\langle B^{2}\right\rangle$, where $B$ is the total field, and \langle\rangle denotes a surface integration via the same operation as discussed for the overlap integral above. Thus,

$$
T \propto\left\langle\left(B_{\mathrm{EN}}+B_{\mathrm{EA}}+B_{\mathrm{I}}\right)^{2}\right\rangle .
$$

As by definition $\left\langle B_{\mathrm{EN}} B_{\mathrm{I}}\right\rangle=0$ and $\left\langle B_{\mathrm{EN}} B_{\mathrm{EA}}\right\rangle=0$, this gives

$$
T \propto\left\langle B_{\mathrm{EN}}^{2}\right\rangle+\left\langle\left(B_{\mathrm{EA}}+B_{\mathrm{I}}\right)^{2}\right\rangle .
$$

However, $B_{\mathrm{EA}}$ can be obtained from the empirically measured I-coil field that gives optimal error field correction on DIII-D, $B_{\text {Ioptimal }}$, with $B_{\mathrm{EA}}$ being equal and opposite to that field, $B_{\mathrm{EA}}=-B_{\text {Ioptimal }}$. This can be obtained from an I-coil phase 
scan, where I-coil fields of different toroidal phases are ramped to induce a mode. Mode formation is deemed to happen at the same total field, and thus the different I-coil current levels at mode formation can be used to infer the equivalent intrinsic error field size and phase as a vector in the $R-\phi$ plane. This thereby allows the identification of the optimal I-coil toroidal phase and magnitude for intrinsic error correction. Such optimization is confirmed empirically with density rampdown studies (optimal error correction leads to lower density access) [12].

$B_{\mathrm{EN}}$ can also be determined experimentally, using density ramp-down studies: as the minimum density to avoid triggering modes is found to be proportional to the size of applied field [3], a non-zero $B_{\mathrm{EN}}$ implies a non-zero density limit even with optimal I-coil correction. Thus

$$
n_{\text {lock }} \propto\left[\left\langle B_{\mathrm{EN}}^{2}\right\rangle+\left\langle\left(B_{\mathrm{I}}-B_{\text {Ioptimal }}\right)^{2}\right\rangle\right]^{0.5},
$$

and the ratio of the magnitudes of $B_{\mathrm{EN}}$ to $B_{\text {Ioptimal }}$ can be deduced from the fractional fall in density limit between zero I-coil and optimal error correction (eliminating terms in the above equation by its application in the two conditions of no and optimal correction). This density ratio is found to be $-38 \%$ for the present device configuration with similar shape and $q_{95}$ plasmas. This gives a total torque and equivalent field magnitude that captures the empirical trends with density and I-coil current amplitude of the form:

$$
T \propto B^{2}=\left(B_{\text {I }}-B_{\text {Ioptimal }}\right)^{2}+0.62 B_{\text {Ioptimal }}^{2} .
$$

Thus the effective applied field is expressed in terms of the known I-coil fields at time of measurement and from the known empirically determined optimal field for intrinsic error correction. In terms of the resonant boundary field discussed above, this leads to a magnitude for $B_{\mathrm{EN}}$ of $1.3 \mathrm{G}$-this is the lowest equivalent level of the total $3 \mathrm{D}$ field, $B$, achievable on DIII-D under optimal error field correction applied by the I-coils.

It should be noted that there is something of a contradiction in the above approaches. For the toroidal mode of greatest concern, $n=1$, which couples most strongly to $q=2$, the ideal response model identifies a single 'dominant' poloidal eigenstructure for $q=2$ interaction (which is in fact a particular combination of external poloidal harmonics). This suggests that optimizing phase and amplitude of a correction field with a single toroidal array of correction coils, and any fixed poloidal spectrum, should achieve near perfect correction [11]. However, the DIII-D operational experience shows such optimization typically achieves less than a $50 \%$ reduction in density limit $[6,12]$, despite predictions of much better correction for such cases from the ideal response model [6]. This implies a substantial residual field exists, with additional mechanisms of plasma response, braking or sub-dominant modes to consider that will likely require optimization of more than the one eigenfunction of correction field ("multi-mode error field correction with at least two independent $n=1$ correction coil arrays) - unless the correction field can be applied with the same poloidal and toroidal structure as the intrinsic error field. Despite these concerns over residual fields, the ideal response model does at least provide an effective basis to measure relative strengths and scalings of field thresholds to induce modes, though highlights that if high levels of error

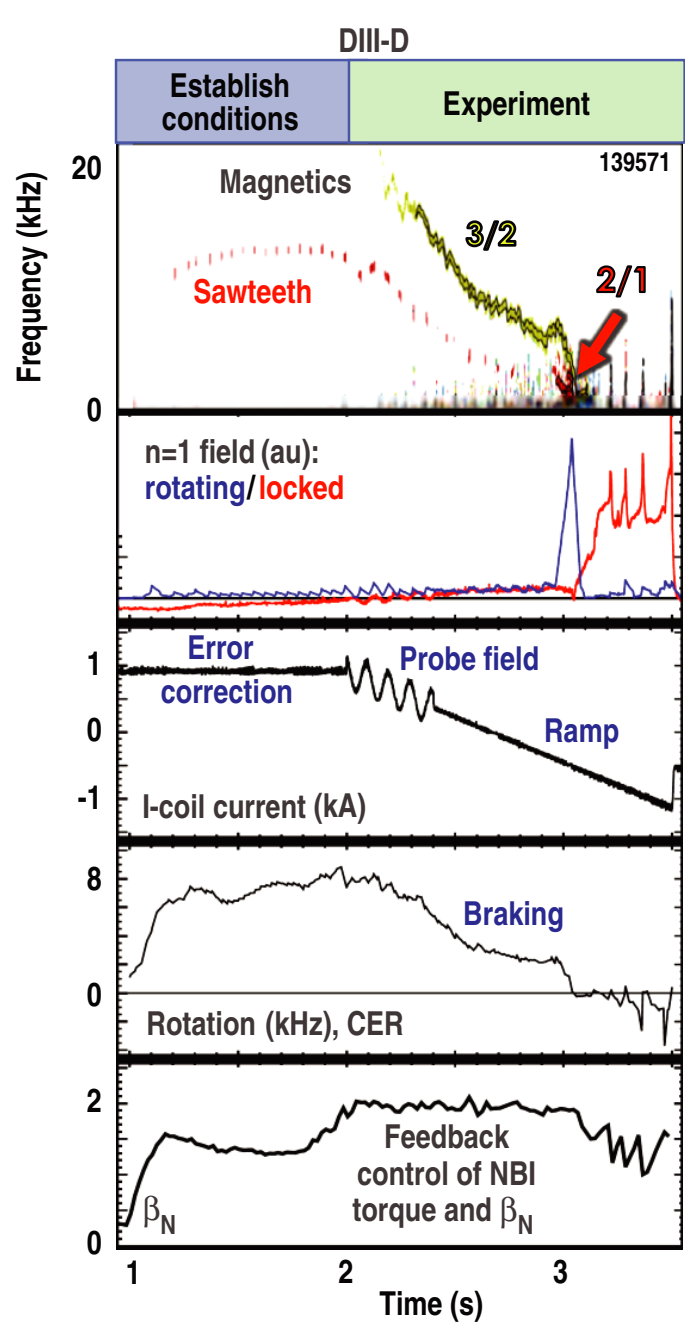

Figure 1. Typical discharge in DIII-D experiment (shot 139571, $q_{95} \sim 4.3$ ). Neutral beam torque (not shown) is maintained constant under feedback control during the time of the field ramp.

correction are needed in ITER, then a more complete multiharmonic treatment must be developed.

\section{Increased 3D field sensitivity in torque-free H-modes on DIII-D}

Experiments were undertaken on the DIII-D tokamak to explore the sensitivity of low torque $\mathrm{H}$-modes to $n=1$ 3D fields, utilizing DIII-D's co- and counter-injecting neutral beams to independently control rotation and $\beta$. A typical discharge is illustrated in figure 1. Plasmas were first established in $\mathrm{H}$-mode at a particular injected torque and $\beta_{\mathrm{N}}$. Heating was started late enough into the discharge for the plasma current profile to relax and sawtooth instabilities to commence, indicative of the presence of the $q=1$ surface, thus producing a fairly reproducible set of initial conditions for the experiment. The generation of a $3 / 2$ mode was also usually observed ahead of a $2 / 1$ mode, providing a further marker to check for reproducible conditions. Torque and $\beta_{\mathrm{N}}$ were adjusted to desired target values for the discharge, and the $3 \mathrm{D}$ field was then ramped, maintaining constant $\beta_{\mathrm{N}}$ and neutral beam torque under neutral beam feedback control. This led to 


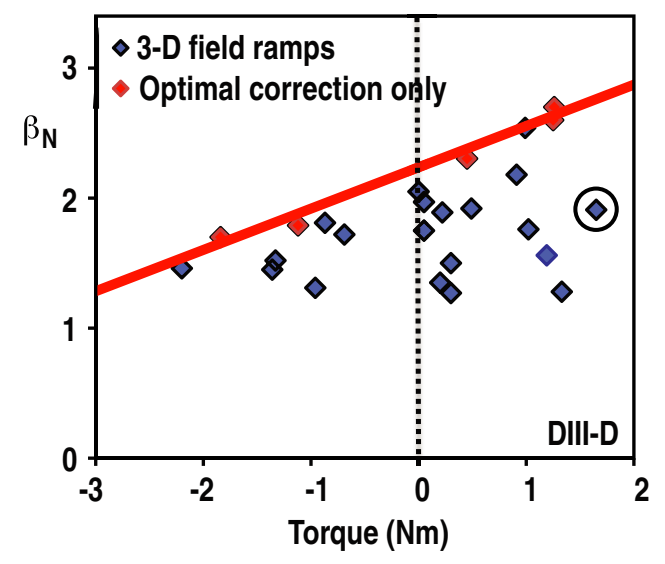

Figure 2. Parameter space explored with DIII-D 3D field ramps (blue), and natural NTM $\beta_{\mathrm{N}}$ limits (red).

a slowing of the plasma rotation and the triggering of a $2 / 1$ tearing mode.

The DIII-D 'I-coils' were used to apply the 3D fields [13]. These are composed of two toroidal rows of six 'picture frame' coils located inside the vessel, above and below the outboard midplane. The fields were applied with a fixed toroidal phase, initially set for optimal correction of the intrinsic error field in DIII-D, before ramping through zero current to progressively increase the net applied field. Connections between the upper and lower rows of coils were offset by $240^{\circ}$ toroidally, which provides the optimum harmonic mix to interact with the $q=2$ surface (to induce modes) or apply error field correction. In the early stages of the field ramp, a low amplitude $10 \mathrm{~Hz}$ toroidally rotating field was also applied to measure the plasma response, and so its linear stability to such driving perturbations.

The plasmas used were based on previous ITER-baselinelike $\mathrm{H}$-modes with matched shape, $\beta_{\mathrm{N}}$ and relaxed profiles [9], though with slightly elevated edge safety factor $\left(q_{95} \sim 4.3\right.$ cf 3.1 in ITER baseline), to facilitate a range of mode studies and avoid the $2 / 1$ modes becoming catastrophic (leading to disruption). These discharges were found to be $\beta_{\mathrm{N}}$ limited by rotating $2 / 1$ tearing modes, identified to be predominantly $\Delta^{\prime}$ triggered (with no significant triggering instability or seeding process-modes often grew up out of the noise). These are sometimes referred to as 'seedless' neoclassical tearing modes (NTMs), where the mode is sustained by the helically perturbed bootstrap current once it reaches large amplitude. The $\beta_{\mathrm{N}}$ limit was also found to fall with decreasing rotation, likewise interpreted as a $\Delta^{\prime}$ effect $[9,10]$. These plasmas therefore represented an excellent target to explore $\Delta^{\prime}$ and rotation dependences in 3D field sensitivity.

Parameters were scanned over a wide range of beam torque and $\beta_{\mathrm{N}}$ values (figure 2) up to the natural tearing $\beta_{\mathrm{N}}$ limitshown in red in the figure, where these $\beta_{\mathrm{N}}$ limit cases were accessed via a $\beta_{\mathrm{N}}$ ramp and no 3D fields were deployed beyond optimal intrinsic error field correction. It should be noted that because of intrinsic torques in the plasma additional to the neutral beam torque, plasma rotations were generally in the co- (i.e. plasma current) direction, reaching relatively high values ( $q=2$ rotation up to several $\mathrm{kHz}$ ) for the most coinjected cases, and approaching zero (but not negative) for the counter-beam dominated cases.

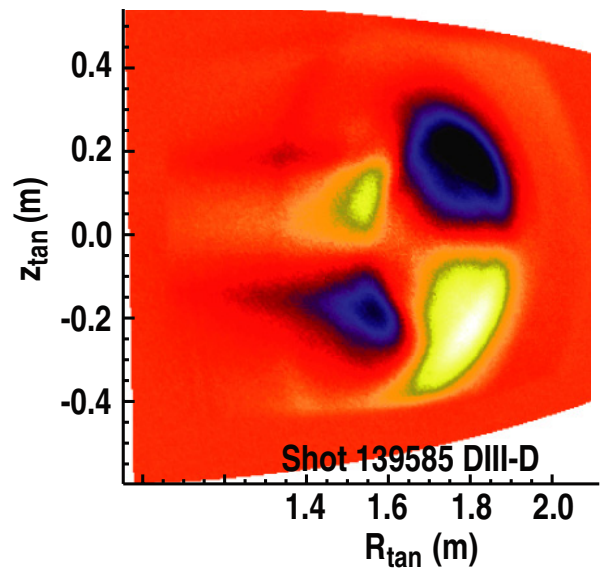

Figure 3. Fourier decomposed fast camera image (tangential view, visible light) of rotating $2 / 1$ mode at $1.8 \mathrm{kHz}$ triggered by $n=1$ field.

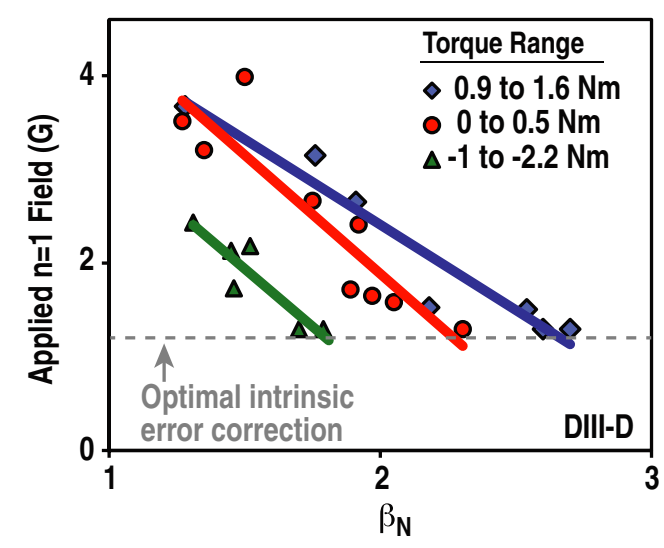

Figure 4. Applied field required to induce $2 / 1$ mode versus plasma normalized $\beta$.

Surprisingly, it was found that modes were generally born rotating, despite the application of a static 3D field triggering the mode at significantly lower $\beta_{\mathrm{N}}$ than that at which the modes would form naturally. Indeed, even with substantial 3D field and low neutral beam torque, the limit came from rotating 2/1 tearing modes, as evidenced by Fourier decomposed fast camera imaging in figure 3 for a case with $7 \mathrm{G}$ of flux averaged boundary field applied and just $0.3 \mathrm{Nm}$ beam torque. Such rotating modes are also evident in the magnetic diagnostics, as illustrated in figure 1, and were observed at nearly all points across the scan. This rotating mode onset indicates a different process from conventional locked mode penetration, with the 3D field acting to change the underlying tearing stability, most likely through rotation braking leading to a decrease in rotation shear.

Field thresholds to trigger these modes are plotted in figure 4 . Note points at the lowest field values, $\sim 1.3 \mathrm{G}$, represent those obtained with optimal error correction as discussed in section 1.1. Results are categorized into three bands of applied neutral beam torque (torque rather than rotation is used as the relevant drive that must be overcome to induce braking). This highlights two key trends. For a given beam torque, as the tearing $\beta$ limit is approached, the required field to induce a mode falls off linearly, reaching the optimum correction level of error field. Also, for a given $\beta_{\mathrm{N}}$, the field threshold falls as torque 


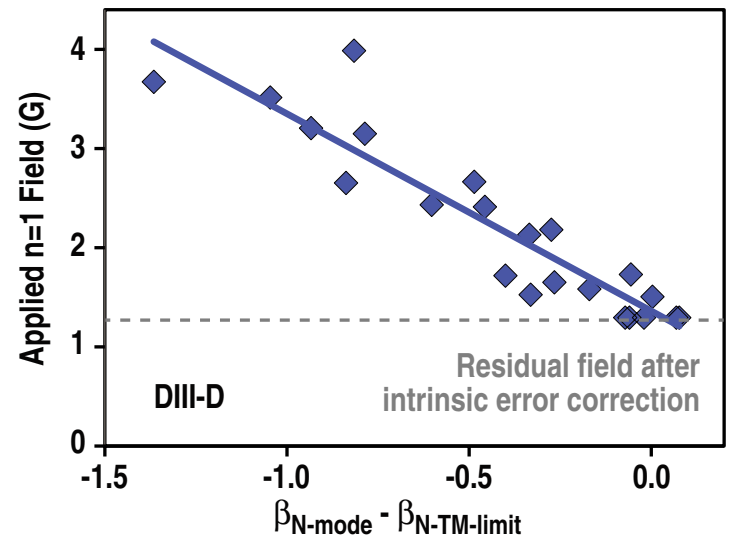

Figure 5. Applied field required to induce $2 / 1$ versus proximity to natural tearing $\beta_{\mathrm{N}}$ limit.

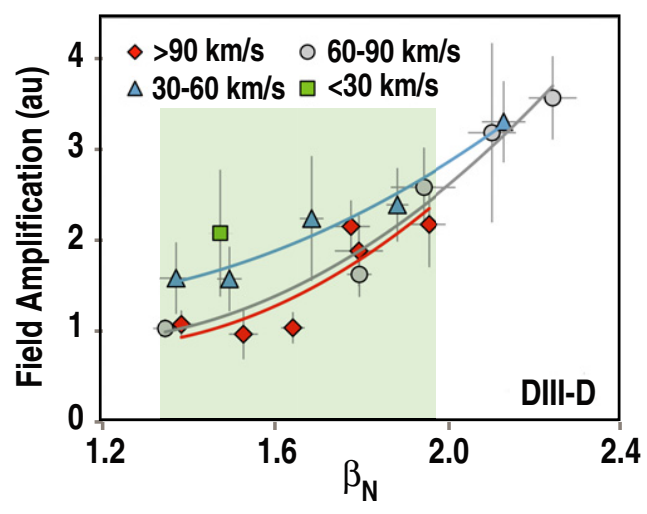

Figure 6. Plasma response to probing field with quadratic regression lines highlighting differences for different rotation ranges. Plasma response is the ratio of measured field at the saddle loop sensor (excluding applied field) to applied field.

and rotation (which remains in the direction of the plasma current) fall. These trends resemble dependences in error field locked mode thresholds of more advanced and higher rotation discharges [7]. The similar trends here, but in proximity to the rotating tearing mode $\beta$ limit and leading to the onset of rotating modes, suggest the field interaction may also be enhanced by proximity to tearing instability. This appears to make the plasma sensitive to even slight imperfections in the error field correction. Indeed, the threshold can be parametrized purely in terms of this proximity: fitting the optimal error correction tearing $\beta_{\mathrm{N}}$ limits (red points) from figure 2 against torque, a scaling $\beta_{\mathrm{N}-\mathrm{TM} \text {-limit }}=2.22+0.32 T_{\mathrm{NBI}}$ is obtained. It is found that the mode thresholds of figure 4 scale directly with proximity in $\beta_{\mathrm{N}}$ to this empirical limit (figure 5), with no significant additional rotation dependence or improvement to correlation achievable by further fitting. Thus the susceptibility of the plasma to 3D fields appears to depend entirely on proximity to the tearing $\beta$ limit.

These results identify an important operational limit, but to understand how to optimize against this limit it is also important to understand the process. Is the plasma response based purely on proximity to the tearing $\beta_{\mathrm{N}}$ limit? Is rotation important to shield out fields? Is tearing stability being decreased or is the 3D field being amplified? Insight is gained from magnetic probing with a small $10 \mathrm{~Hz}$ field, performed at each scan point before the field was ramped up to larger values. This shows (figure 6) plasma response increasing substantially with $\beta_{\mathrm{N}}$-thus less external field is needed to cause braking [7] and trigger modes at high $\beta_{\mathrm{N}}$ because the plasma amplifies the field more. This can be interpreted as an ideal-MHD-like response, with the kink mode driven more readily at higher $\beta_{\mathrm{N}}$ [14]. However, at constant $\beta_{\mathrm{N}}$ with values $<2$, the plasma response also rises as rotation (measured in the vicinity of the $q=2$ surface) falls.

This rotation dependence highlights a possible resistive response-as shielding weakens at low rotation, this allows greater tearing of the 'suppressed' island at the rational surface. Proximity to the natural tearing unstable $\beta_{\mathrm{N}}$ limit may also be an important part of this response, as rotation scans away from this limit show no such effect [15]. $\beta_{\mathrm{N}}$ may influence this process in two ways: increasing $\beta_{\mathrm{N}}$ raises the plasma ideal response, amplifying the field, and therefore the local field at $q=2$, so potentially increasing the tearing response. But increasing $\beta_{\mathrm{N}}$ is also known to lower $\Delta^{\prime}$ stability [16], thereby potentially further increasing plasma resistive response in the situation of weak shielding. From the data, it is hard to see whether and which of these specific $\beta$ related effects are part of the process-thus we explore them further with modelling in the next section. Nevertheless, based on the experimental observations, we hypothesize that increases in the plasma resistive response to $3 \mathrm{D}$ fields, that occur as $\Delta^{\prime}$ stability and rotation shielding are weakened, give rise to a lowering of $3 \mathrm{D}$ field thresholds. Thus raising rotation and improving tearing stability through the current profile may be key factors in optimizing plasma resilience to 3D fields.

Analysing the trends in plasma response more carefully, it appears that both ideal and resistive responses are playing a role. Figure 6 already shows that the effect is likely not to be purely ideal, due to the additional rotation dependence. However, in contrast to the behaviour of the error field threshold discussed in figure 5, the plasma response to probing fields does not correlate well with proximity to the fitted tearing mode $\beta_{\mathrm{N}}$ limit (figure $7(a)$ ). Instead we see dependences on $\beta_{\mathrm{N}}$ and rotation (figures $7(b)$ and $(c)$ ), with a regression scaling of $\beta_{\mathrm{N}}^{2.25} \Omega^{-0.47}$ giving a better correlation than fits to any one parameter. This suggests that the behaviour is not well represented by any single process, such as ideal response (which would principally give a $\beta_{\mathrm{N}}$ dependence) or a resistive response (which might depend on rotation or simply tearing limit proximity). Instead both ideal and resistive effects will likely be playing a role.

\section{Understanding the resistive response through modelling with MARS-F}

To explore these interpretations of a plasma ideal and resistive response further, modelling was executed using the MARS-F code $[17,18]$, a single fluid code that solves the linearized resistive MHD equations for three-dimensional perturbations in two-dimensional toroidal geometry. This code can calculate both the ideal and the resistive response to applied fields, though it should be noted that being in the linear regime, it does not include non-linear amplification effects, as might occur if the torque associated with a resistive response 

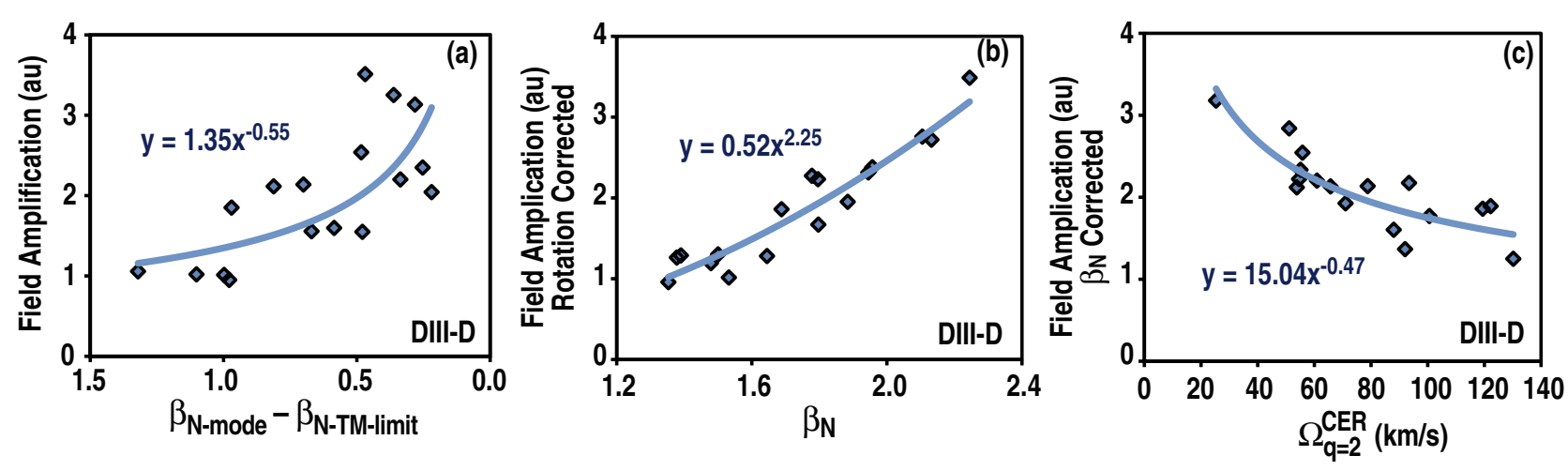

Figure 7. Plasma response, as defined in figure 6 , plotted $(a)$ versus proximity to tearing $\beta_{\mathrm{N}}$ limit, or as a function of $\beta_{\mathrm{N}}(b)$ and rotation $(c)$ correcting for other parameter using $2 \mathrm{D}$ regression fit.

changes the phase of that response, leading to increased tearing and potentially further amplification and torques. Nevertheless, MARS-F can act as a good indicator to test the principles of whether a resistive response might start to develop for the plasma used here, and how this response might depend on plasma parameters. This is the goal of this study.

MARS-F modelling was executed for a representative case-shot 139571, circled in figure 2-near the ITER baseline $\beta_{\mathrm{N}}$, at a value of 1.9 , but with relatively positive neutral beam injection (NBI) torque and high rotation. Equilibrium parameters were fed in from a kinetic EFIT, taking realistic core and pedestal pressure profiles and associated bootstrap current, and motional Stark effect (MSE) data to constrain the core current profile. It should be noted that pedestal data were not of the highest quality in these discharges, so the pedestal taken tended to be more typically representative of this type of discharge, rather than a precise representation of this particular discharge. (This was considered acceptable, as the point of this modelling was to establish the principles of the dependences, rather than a detailed numerical match.) Rotation, density and temperature profiles were fed into the MARS-F reconstruction and resistivity profile calculated. A smoothed version of the DIII-D wall was employed, and the experimental configuration of I-coils was used to calculate plasma response. Parameters were then scanned about the experimental values to explore underlying effects and their variation.

For the high rotation base case, as expected, MARS-F indicated significant amplification (factor $\sim 3$ ) for a range of harmonics ( $m=1-6$ ) of applied field across the plasma (figure 8(a)), while resonant components were shielded out at rational surfaces. Rotation was then scanned down from the experimental value, with other parameters held fixed, to look for changes in the plasma response, and the hypothesized resistive response. It was found (figure $8(b)$ ) that plasma response only changed modestly with rotation at most radiiconsistent with the ideal response depending weakly on rotation. However, the local response at the rational surface demonstrated a much more dramatic effect, with the shielding starting to break down and significant tearing starting to occur. The break down of shielding is made clearer in figure $8(c)$, where local resonant fields at the relevant rational $q$ flux surfaces are plotted against a radial ordinate. It can now be seen that the plasma shielding response decreases significantly with lower rotation, causing the total resonant field shown in the figure to rise from near zero (high shielding, strongly suppressed island) towards the vacuum field (no shielding, significant tearing). The size of the suppressed island perturbation from MARS-F, compared with vacuum field, is consistent with estimates on a theoretical basis [19]: this ratio should approximate to $1 /\left(\omega \tau_{\text {tear }}\right)$, where $\omega$ is the $q=2$ rotation in $\mathrm{rad} \mathrm{s}^{-1}$ and $\tau_{\text {tear }}$ is the tearing time, which is taken as $\tau_{\mathrm{R}}^{3 / 5} \tau_{\mathrm{A}}^{2 / 5}$. Using typical numbers for this discharge, $\tau_{\mathrm{R}}=5 \mathrm{~s}, \tau_{\mathrm{A}}=0.5 \mu \mathrm{s}, w=44 \mathrm{krad} \mathrm{s}^{-1}$, a value of $\omega \tau_{\text {tear }} \sim 350$ is obtained (or $\sim 800$ using the more sophisticated treatment of equation (9) in [20]), comparable to the factor 930 suppression observed for $q=2$ in figure $8(c)$. Thus it appears that access to the low rotation regime opens the door to this additional resistive response. It should be noted that in the linear modelling and theory discussed here, the rotation decrease required to reduce shielding in the MARS calculation is greater than that found in the experiment. However, a quantitative comparison would require a non-linear treatment and this may lead to an increased calculated response as rotation is lowered, potentially improving the match.

As the observed 3D field effects occur in proximity to the tearing mode $\beta$ limit, and it has been previously argued [9] that this limit principally arises as a $\Delta^{\prime}$ driven mode, it is instructive to check for sensitivity to parameters governing this effect. Indeed, this is highly pertinent to the mechanism as the original Fitzpatrick theory [1] predicts a dependence of the plasma response on $\Delta^{\prime}$. Thus studies of $\beta$ and current profile variation were undertaken. It is found that access to low rotation breaks the usual linear dependence of plasma response on $\beta$. In figure $9(a)$, the peak value of the calculated $m=2$ response (peak of curves in figure $8(a)$ ) is taken as a measure of the global response, and plotted as $\beta$ is varied relative to the experimental value, for three rotation levels. This shows peaks and increases in response for the low rotation cases, which can be understood from figure $9(b)$ as an increase in the calculated local resonant surface tearing response, which depends on $\beta$. It is particularly interesting that this local response of figure $9(b)$ appears to correlate closely with a significant rise in the global response measured well away from this surface in figure $9(a)$, suggesting some possible coupling of resistive and ideal effects. Also of interest, the peaks at low rotation seem to occur at similar $\beta_{\mathrm{N}}\left(\sim 1.7\right.$ corresponding to $x=\beta / \beta_{\text {crit }}=0.9$ in the plots) to the natural tearing $\beta_{\mathrm{N}}$ limits in low rotation 
MARS-F Simulation of DIII-D
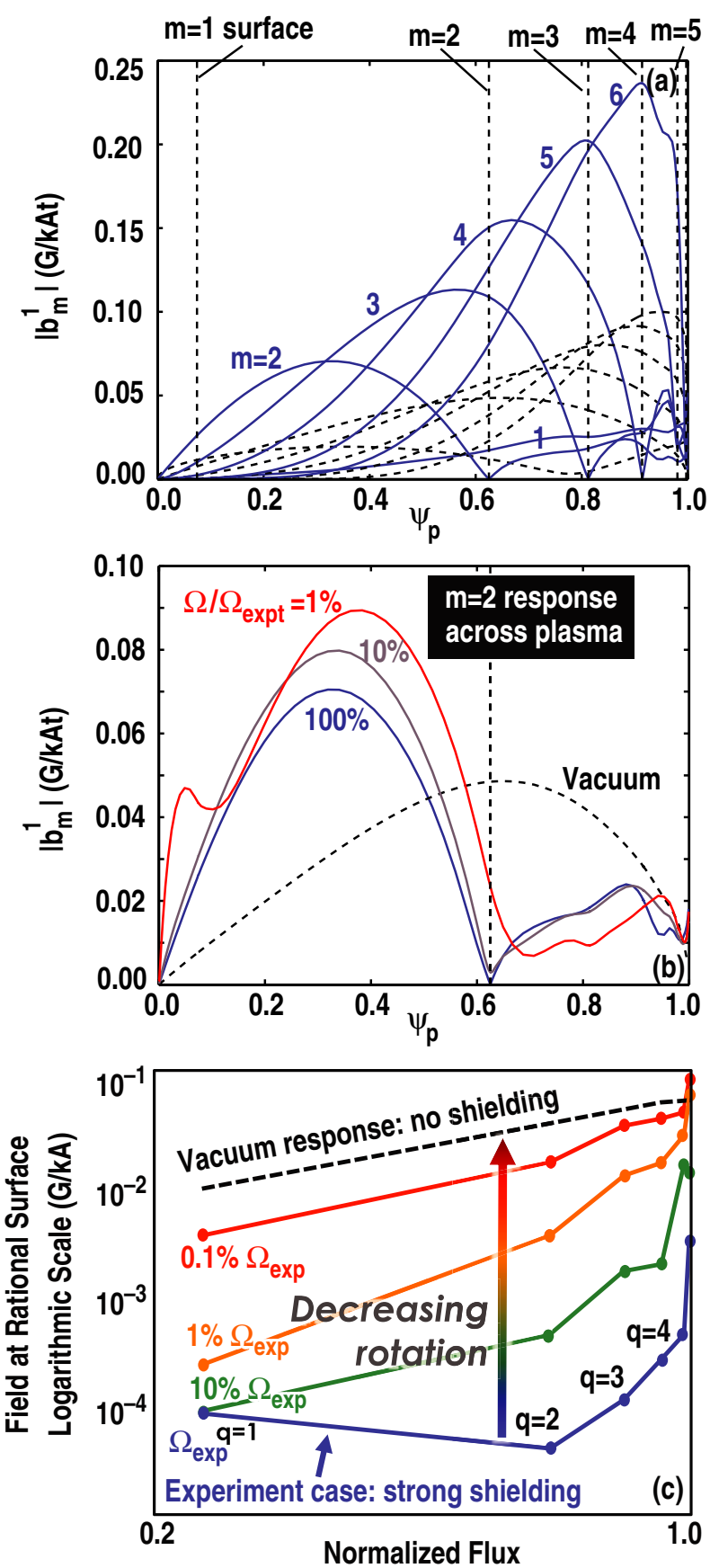

Figure 8. (a) MARS-F calculated $n=1$ plasma response $\left|b_{m}^{1}\right|$ (solid line is vacuum + plasma response, dashed line is vacuum) to the range of poloidal harmonics in the I-coil field for the high rotation base case, 139571. (b) Calculation of $m=2$ plasma response to I-coil field by MARS-F for DIII-D shot 139571 at three values of plasma rotation relative to experimental profile. (c) Applied field + plasma response at rational surfaces calculated by MARS-F, based on parameters and profiles of DIII-D shot 139571 (circled in figure 2), but with rotation profile scaled as indicated: the shielding response is lost as rotation is lowered, indicating development of the tearing response.

(negative torque) cases, where there is a very high error field sensitivity for inducing modes. Indeed this may explain some of the $\beta_{\mathrm{N}}$ dependence observed in the tearing mode $\beta$ limits.

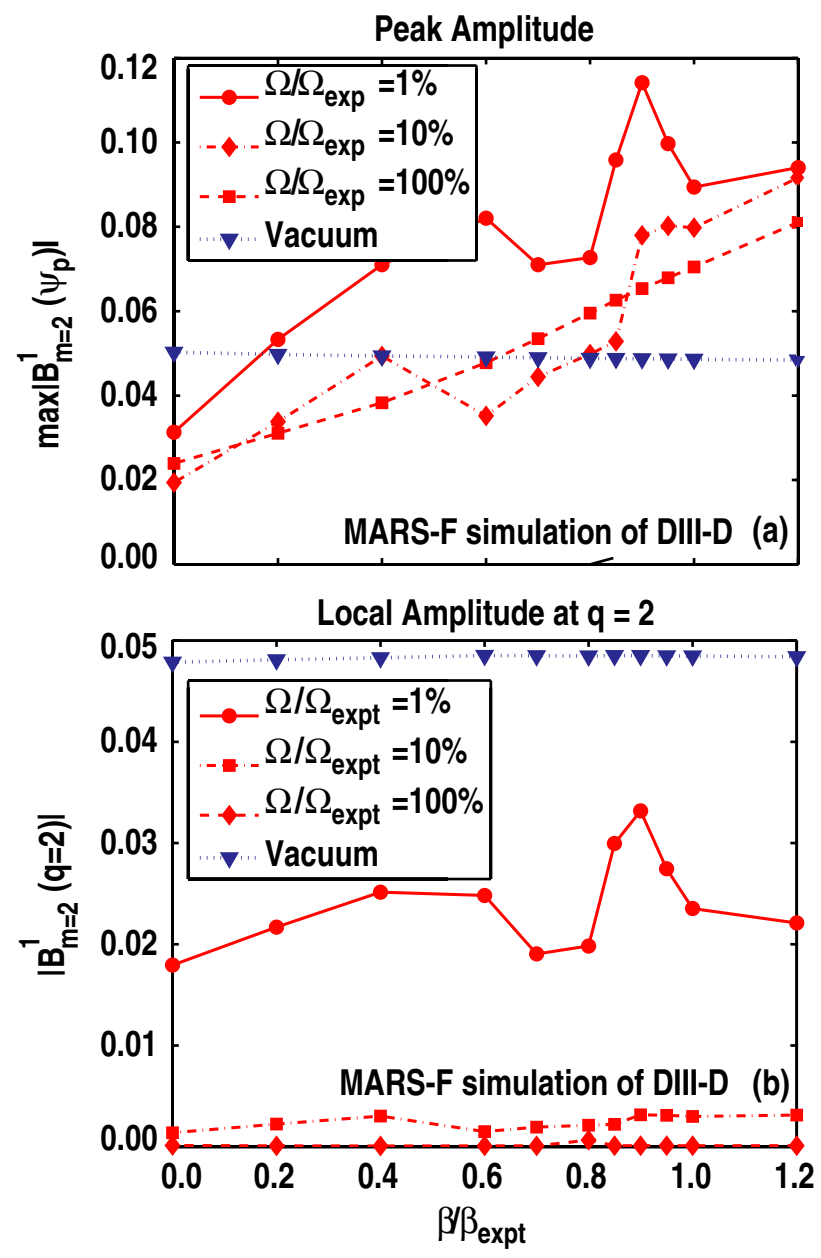

Figure 9. (a) Peak value of calculated plasma response (across all radii) to $m=2$ field plotted as $\beta$ varied for three values of rotation (b) Total calculated field at $q=2$ surface plotted as $\beta$ varied for three values of rotation.

Finally, to explore and better understand the hypothesized role of $\Delta^{\prime}$ in the stability, the plasma response was evaluated in MARS-F for a systematic hypothetical variation of the parallel current density near the $q=2$ rational surface. An arbitrarily chosen functional form was used, simply to test whether a sensitivity is present:

$J_{\|}^{\text {new }}=J_{\|}-a J_{\| \mathrm{q}=2}^{\prime}\left(x-x_{\mathrm{q}=2}\right) \exp \left[-\left(\frac{x-x_{\mathrm{q}=2}}{0.05}\right)^{2}\right]$,

with $x$ being the square root of normalized poloidal flux, while the parameter ' $a$ ' in equation (1) was varied from -2 to +4 to change the current and safety factor profiles as indicated in figure 10. The corresponding global and local measures of how the plasma response to 3D fields changes with current profile are shown in figure 11. As with the $\beta$ dependence, it is found that at high rotation there is little change in the response with current profile variation, because the response remains predominantly ideal in nature. But the resistive response at low rotation leads to a dependence on the current profile, with the local $q=2$ response (figure $11(b)$ ) found to increase as current is distributed outside of $q=2$ and current gradients at $q=2$ reduce and then reverse. Naively, the current gradient might 


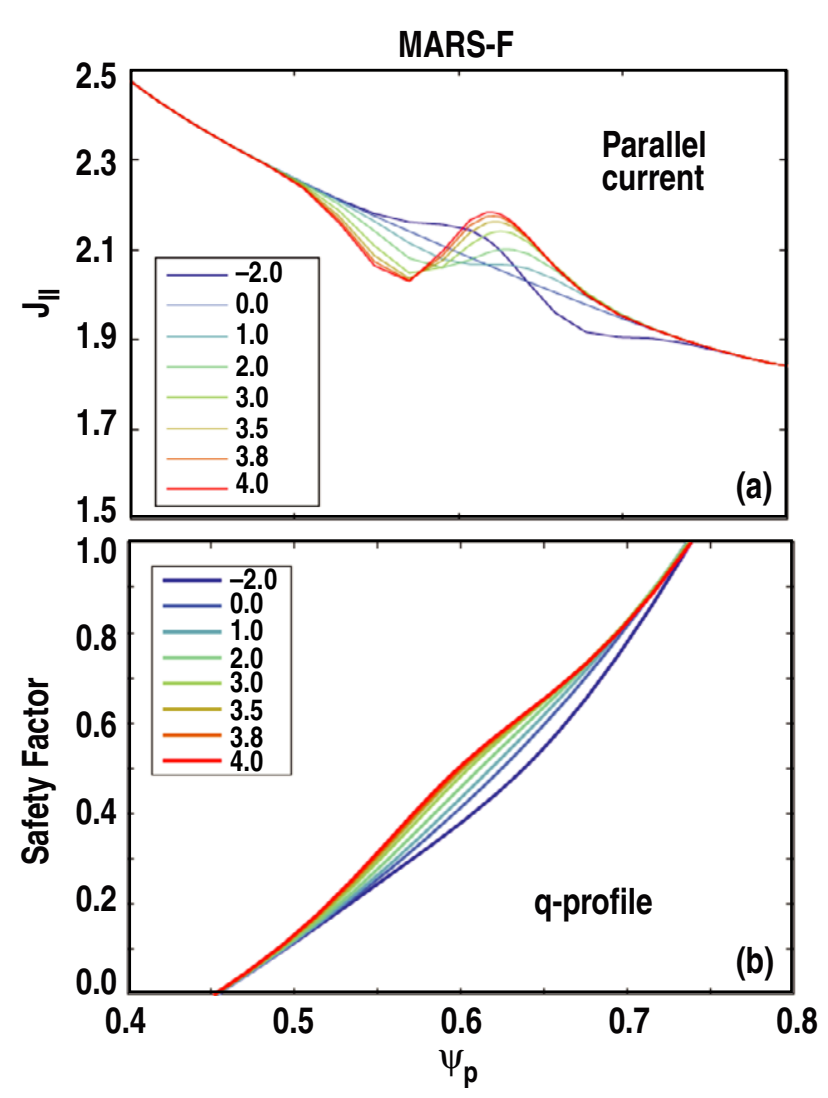

Figure 10. Hypothetical variations in current profile explored in the vicinity of $q=2(a)$ and associated change in $q$ profile $(b)$. Key indicates variation in size of the current profile perturbation parameter ' $a$ ' as described in the text.

be considered to drive tearing instability, thus a local reduction might be thought to be stabilizing. However, an analytic computation of $\Delta^{\prime}$ [21] shows that, in fact, tearing stability is maximized near to zero current gradient (figure 12(a), where more negative $\Delta^{\prime}$ is more stable). Therefore, the changes in predicted plasma response, which is expected to scale inversely with $\Delta^{\prime}[22]$, appear broadly consistent with the $\Delta^{\prime}$ variation (with an offset possibly explained by other terms in the theory). It should also be noted that an empirical model of the effect of local flow shear on $\Delta^{\prime}$ in equation (5) of [10] shows how $\Delta^{\prime}$ can become insensitive to local magnetic shear with sufficient flow shear, thus giving further insight into the rotation dependence. Nevertheless, the situation is likely more complex, noting that this small current profile modification close to $q=2$ also leads to large changes in the global plasma response, represented in figure 11(a). This is highlighted further in figure $12(b)$ where the $m=2$ response across the whole plasma is observed to vary substantially. Thus again, the ideal and resistive responses appear coupled and potentially mutually amplifying.

These modelling observations clearly merit a much deeper study into the origins and dependences of the resistive response and the relation to global plasma response. This is beyond the scope of this work. The key points here are that low rotation opens the door to a resistive response of the plasma to $3 \mathrm{D}$ fields, and that this response appears to depend on the main parameters related to tearing mode stability, such as $\beta$ and the current profile. Thus it is the combination of low rotation and weak tearing stability that maximizes the resistive response
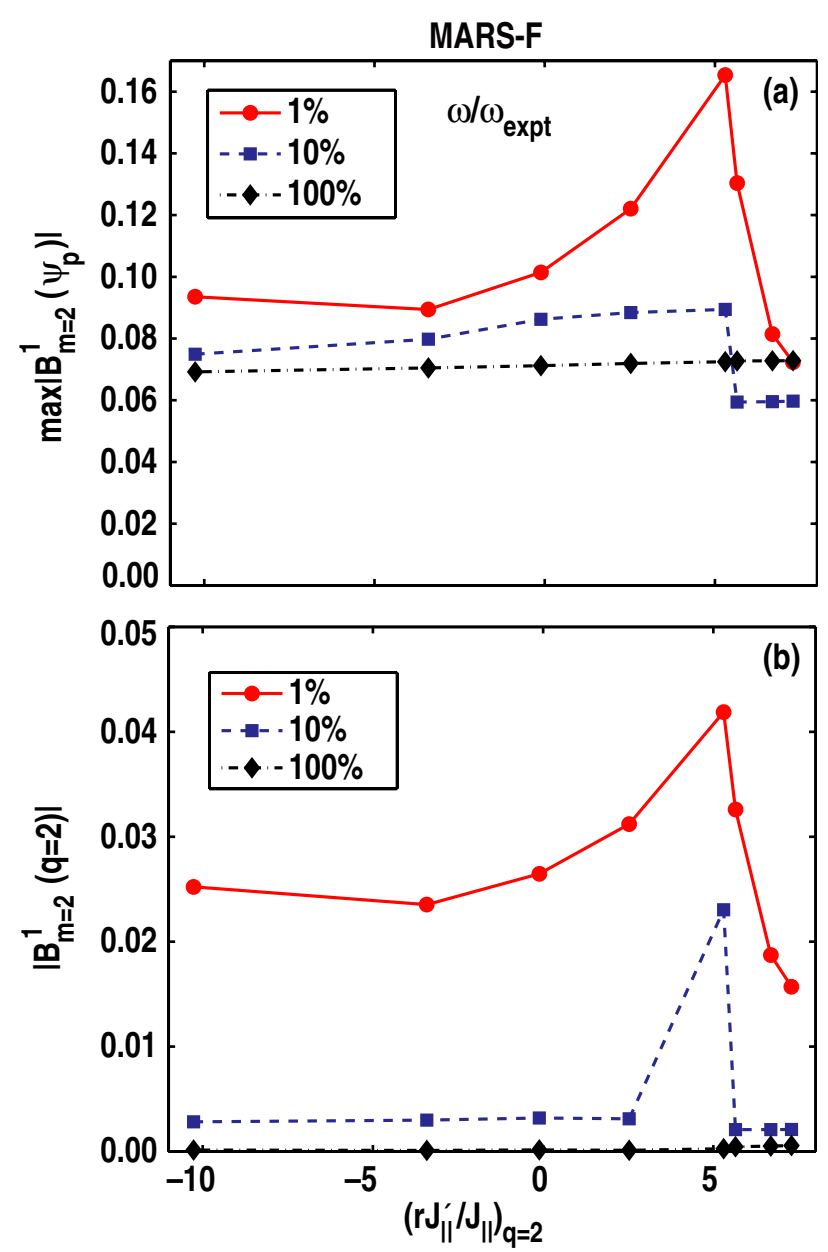

Figure 11. Change in plasma response as current profile and rotation varied hypothetically as in figure 10 , measured in terms of peak amplitude of the $m=2$ response across the plasma $(a)$, and local resonant field at the $q=2$ surface $(b)$. Response is plotted against current gradient (which has a negative value for the base case).

to 3D fields, while increased $\beta$ can further amplify fields or decrease proximity to tearing limit. These basic facts align with the original theoretical concepts on 3D field interactions of [1], and with the experimental observations reported here of rotation and $\beta$ driven dependences in the plasma response and mode-trigger threshold. In particular, the MARS-F modelling provides a qualitative validation of the proposed mechanisms in section 2 , of a breakdown in plasma shielding leading to a resistive response, which in turn leads to a dependence of $3 \mathrm{D}$ field effects on proximity to intrinsic tearing stability limits.

\section{Understanding the interaction of 3D fields with the tearing $\beta$ limit on NSTX}

To provide a consistent basis for extrapolating tolerable error field thresholds, it is important to understand the interaction of the 3D field with the intrinsic tearing mode instability. Is a resonant field needed? How does braking alter the rotating tearing mode stability? How does this physics connect with the conventional penetration to locked mode?

NSTX is a useful tool to explore these issues, as its time evolving profiles can help deconvolve rotation from rotation 

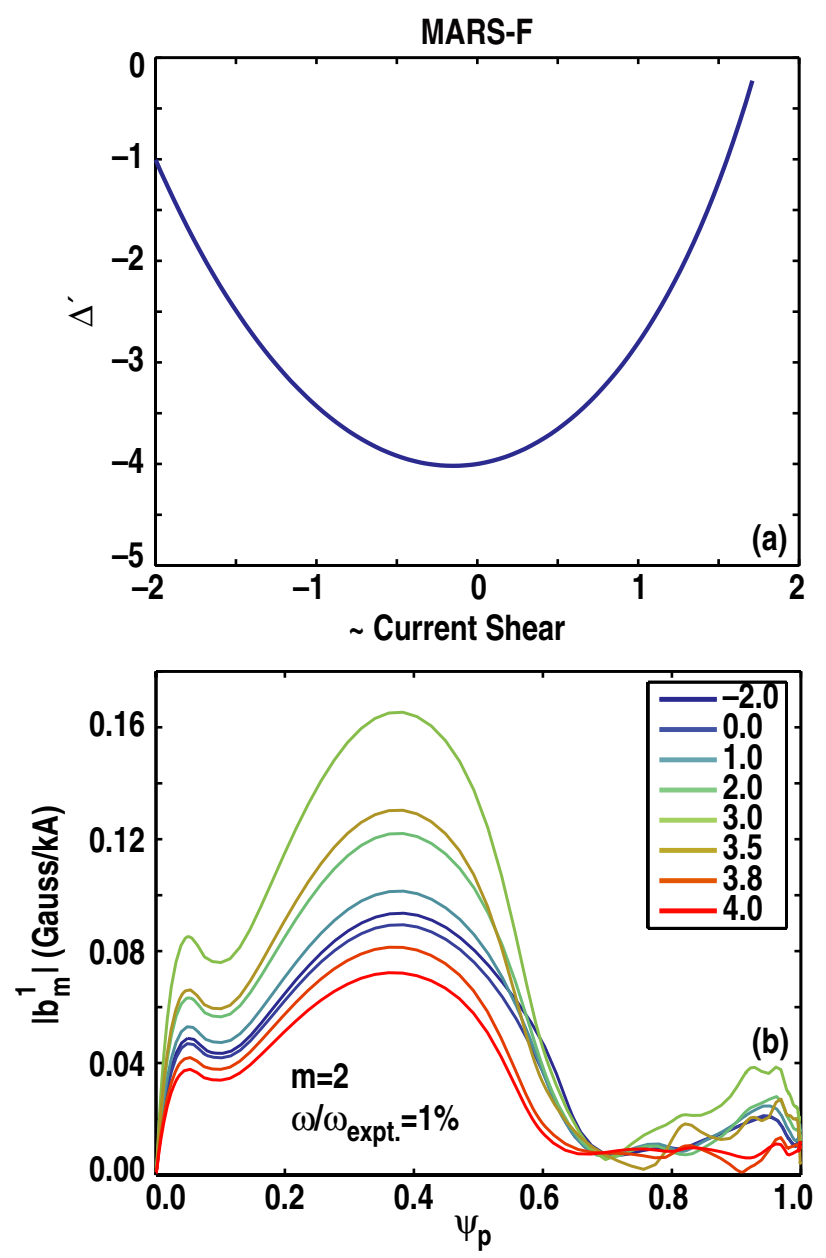

Figure 12. (a) Analytic calculation of $\Delta^{\prime}$ variation [19] for hypothetical current profile changes considered here, plotted against an approximate calculation of the current density gradient. (b) $m=2$ response of plasma to I-coil versus normalized flux for the range of current profiles discussed.

shear effects in NTM stability [23], and, like DIII-D, can apply both $n=1$ and $n=3$ fields to explore questions of resonance. Here plasmas were established in $\mathrm{H}$-mode with steadily rising $\beta$ as profiles evolved, with central safety factor falling towards unity (figure 13). At the start of the discharge, 3D fields were applied principally to compensate a known $n=3$ intrinsic error field. Then at $400 \mathrm{~ms}$ an additional field ramp was applied comprising of $n=1$ or $n=3$ fields, or some fixed ratio thereof. Inter-shot lithium evaporation was used to minimize edge localized modes (ELMs), removing these as potential triggers of the modes.

The resulting mode thresholds are plotted in figure 14 in terms of local NTM bootstrap drive $[25,26]$ (as profiles are evolving, $\beta_{\mathrm{N}}$ is no longer a good proxy for this). Rotation shear is normalized for inverse Alfvén time and magnetic shear scale length, as proposed in [10]. These data show a weak but significant trend with the rotation shear based parameter, while no significant trend in rotation, indicating the $3 \mathrm{D}$ field acting through rotation shear on underlying tearing mode stability, in a similar manner to observations of trends in natural NTM $\beta$ limits on NSTX [22] and elsewhere [9, 10].

The action of these fields on the plasma rotation is explored in figures 15 and 16 . Here modes were accessed with different

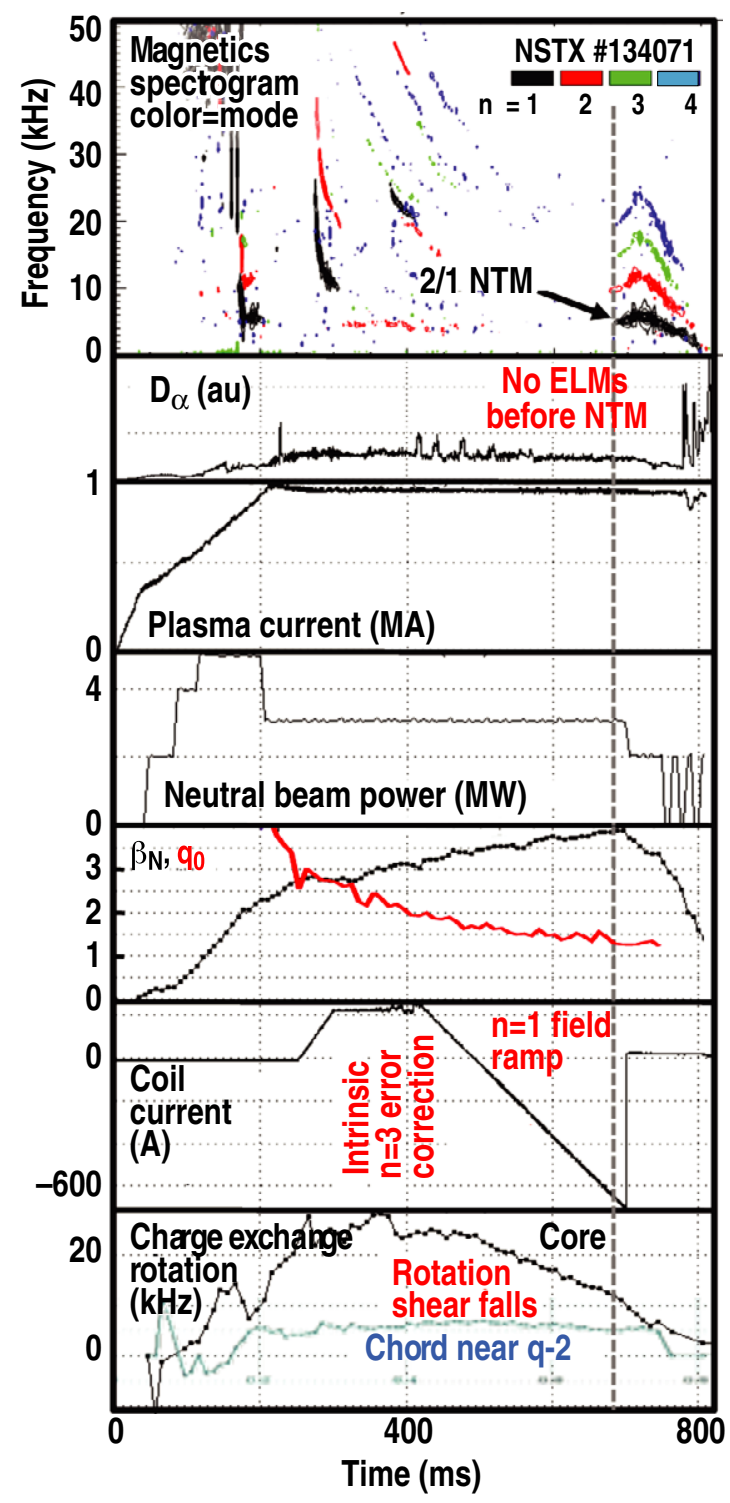

Figure 13. Typical NSTX time evolution discharge.

levels of field by varying the field ramp; a faster ramp would trigger the mode earlier in the discharge, at lower NTM drive but with increased applied field. As it is hard to quantify $n=1$ and $n=3$ fields on the same basis, we use the simplest measure of amplitude possible: the current in the coils. Figure 15 shows that for both types of field there is a transition from rotating to locked mode formation at similar levels of $n=1$ or $n=3$ applied field. Exploring the rotation response through the birth frequency of the triggered mode (figure 16), we find both types of field exhibit progressive and similar magnitude braking, with a best fit taking the form $\Omega_{21}=6958-\left(2.26 I_{\mathrm{n}=1}+2.52 I_{\mathrm{n}=3}\right)^{2}$. These data suggest the main action of the fields is through braking, either by changing the rotating mode's stability, accessing it at lower drive through decreased rotation shear, as in figure 14, or by reaching a critical $50 \%$ of natural rotation level, when the plasma transitions directly to a locked mode.

The sharp rotation threshold with $n=3$ field is in fact surprising, because this field is non-resonant and should not 

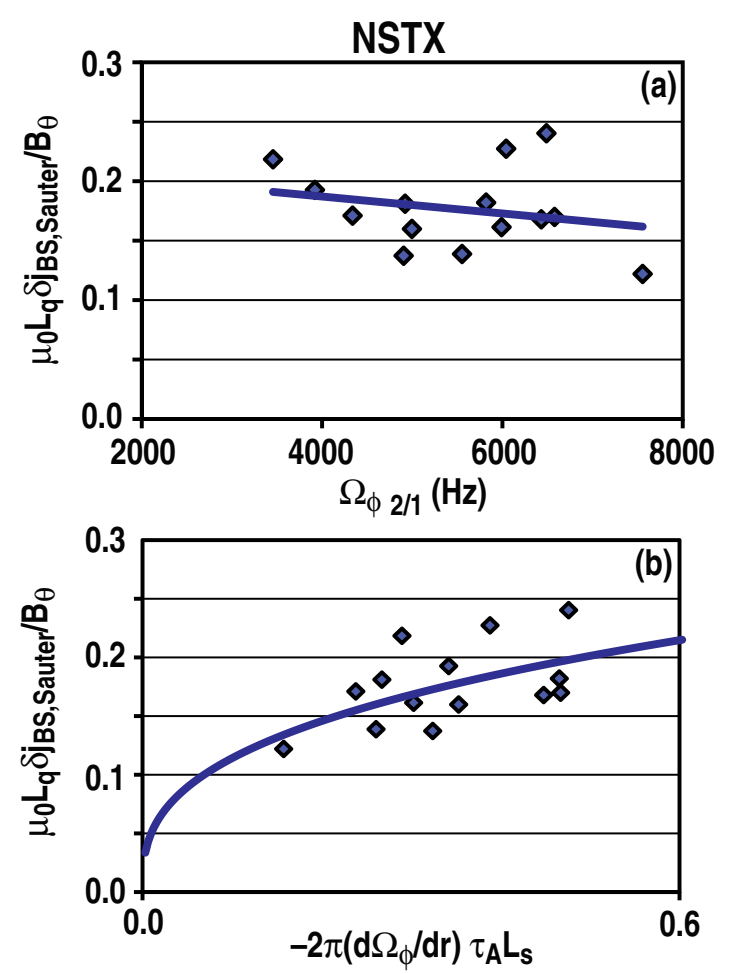

Figure 14. Local $q=2$ electron bootstrap drive for NTM stability as defined in [18] at NTM onset versus rotation $(a)$ and rotation shear $(b)$ on NSTX as $n=1$ and $n=3$ fields varied.

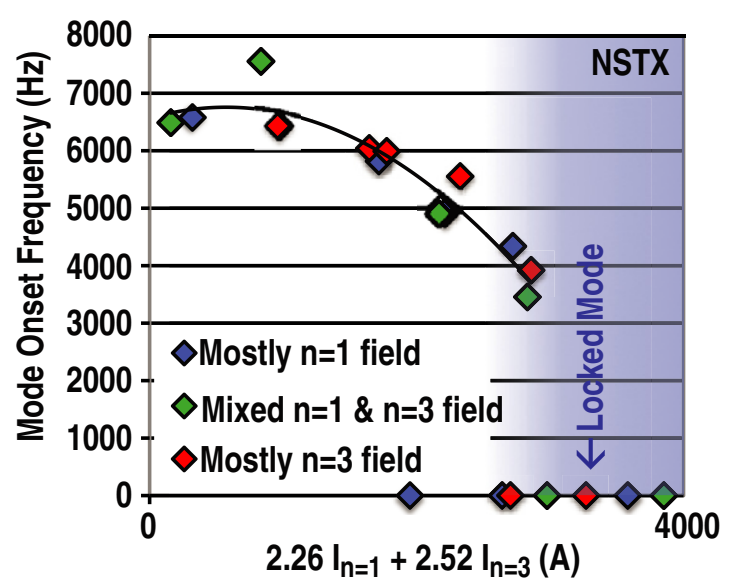

Figure 15. Occurrence of modes with $n=1$ and $n=3$ fields.

drive a localized $q=2$ response that leads to a loss of torque balance and sudden penetration as expected for $n=1$ fields (section 1). Such non-resonant fields are usually observed to brake the plasma through the effects of neoclassical toroidal viscosity (NTV) to lower rotation without the triggering of a locked mode [24]. An explanation may lie in the proximity of these plasmas to tearing instability coupled with the lowering of rotation by $n=3$ fields-this potentially increases the sensitivity of the plasma to any residual low amplitude $n=1$ field enabling the conventional penetration process, a process also observed in [7]. The similarity of $n=1$ and $n=3$ braking effects might also be explained if we consider that the $n=1$ field will contain both resonant and non-resonant components. As non-resonant braking increases with plasma rotation, while

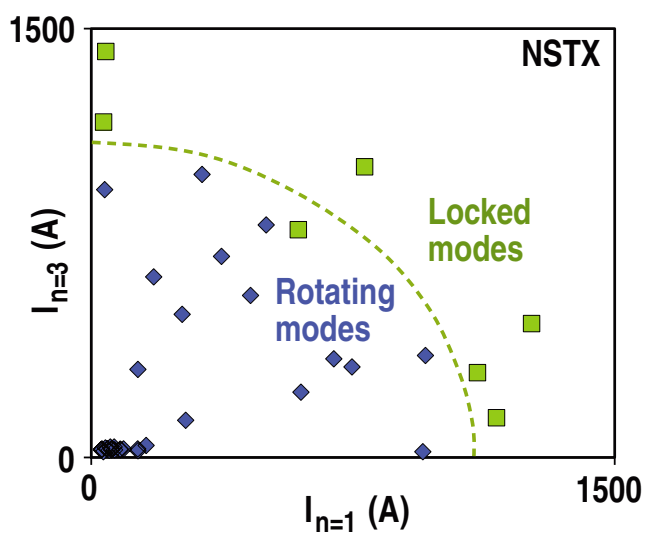

Figure 16. Braking effect of $n=1$ and $n=3$ fields based on optimal correlation 2D fit as described in the text.

resonant braking falls, it is possible that in the high rotation plasmas used here, the $n=1$ field acts predominantly through non-resonant braking, as with the $n=3$ fields, until rotation is lowered sufficiently for resonant effects to take over.

From this analysis, a key point emerges: the main action of $3 \mathrm{D}$ fields on tearing stability for both rotating and locked mode onset is through magnetic braking. Thus, the threshold for mode formation can be considered as one in rotation itself. This is reminiscent of the original Fitzpatrick model [1], which identified a 50\% rotation threshold for mode penetration. Here the criterion might be generalized slightly, as less braking is needed to lower the rotating mode threshold. This perhaps provides a common basis for extrapolating mode field thresholds, at least for given profiles and $\beta_{\mathrm{N}}$, irrespective of the precise onset mechanism-it is the criteria for achieving significant braking to trigger a mode that matters.

\section{Scaling of field thresholds and extrapolation to ITER}

Having established a common mode threshold mechanism based on rotation braking, it is tempting to attempt an error field threshold extrapolation akin to the approach used for ohmic regimes [3]. This utilized a dimensional argument to infer machine size scaling from density and toroidal field dependences. It implicitly included rotation as a hidden variable - a self-generated parameter that plays a key role but is already manifest in the measured threshold scalings, and so included in the extrapolation. However, for H-modes, the origins of plasma rotation are complex, and mechanisms governing rotation may be quite different from ohmic regimes, and so scale differently. Nevertheless, this can be dealt with consistently for ITER, as its relatively low torque injection (predominantly heated by fusion $\alpha$ s and much higher energy beams) allows rotation to again be treated as a self-generated hidden variable, implicit in the scalings. Thus measurements of error field threshold scalings are needed in torque-free $\mathrm{H}$-modes, and have been obtained by utilizing the balanced beam capability of DIII-D with discharges as described in section 2 , fixing $\beta_{\mathrm{N}}$ to the ITER baseline value of 1.8 while density and toroidal field (at fixed $q_{95}=4.4$ ) were scanned from shot-to-shot. 

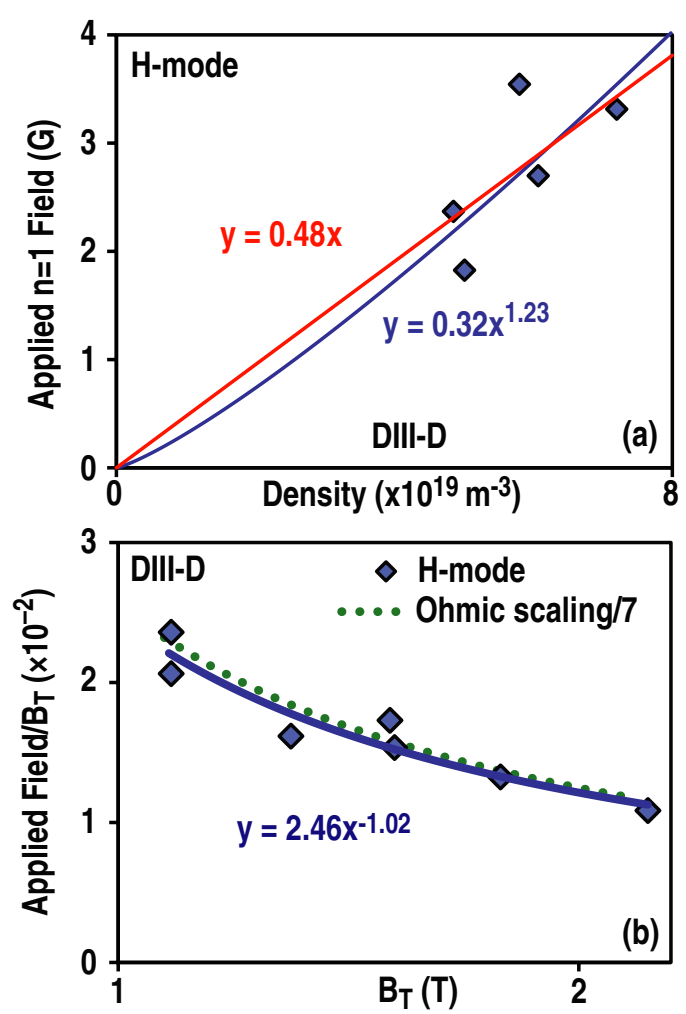

Figure 17. Density ( $a$ ) and toroidal field $(b)$ scaling of $n=1$ mode field threshold in torque-free H-modes on DIII-D at constant $\beta_{\mathrm{N}}=1.8$ and $q_{95}=4.4$. Dotted curve in lower panel represents the ohmic scaling prediction from [3] for the plasma parameters and field calculation methodology used here, though with a further scaling factor of $/ 7$ applied.

The scalings are plotted in figure 17, correcting for slight variations in proximity to $\beta_{\mathrm{N}}$ limit (from slight $\beta_{\mathrm{N}}$ and torque variations) using the approach described for figure 5 . The density scan shows, perhaps, a slightly steeper dependence than the linear scaling found in ohmic regimes, though this is within error bars of a proportional scaling. The toroidal field is almost identical in exponent to the ohmic scalings, except that in order to achieve an overlay, the ohmic scaling has had to be divided by a factor of 7 for the parameters of these plasmas. Using the dimensional constraint of [3] for scaling of the form $B_{\text {pen }} / B_{\mathrm{T}} \sim R^{\alpha_{\mathrm{R}}} n_{\mathrm{e}}^{\alpha_{\mathrm{n}}} B_{\mathrm{T}}^{\alpha_{\mathrm{B}}}$ and assuming linear density scaling (given the sparsity of the data) yields a machine size scaling exponent of $\alpha_{\mathrm{R}}=2 \alpha_{\mathrm{n}}+1.25 \alpha_{\mathrm{B}}=$ 0.725 . Thus the $\mathrm{H}$-mode field threshold scalings are broadly consistent with ohmic scalings, apart from a lower baseline and increased sensitivity in proximity to the tearing $\beta_{\mathrm{N}}$ limit. Combining the dependences with the scalings observed in section 2, an overall scaling for torque-free $\mathrm{H}$-modes is obtained:

$$
\begin{aligned}
\frac{B_{\text {pen }}}{B_{\mathrm{T}}} & =\left[1.72-\left(\beta_{\mathrm{N}}-1.8\right)\right] \\
& \times \frac{\left(n_{\mathrm{e}} / 10^{20} \mathrm{~m}^{-3}\right)(R / 6.2 \mathrm{~m})^{0.725}}{\left(B_{\mathrm{T}} / 5.3 \mathrm{~T}\right)^{1.02}} \times 10^{-4},
\end{aligned}
$$

where the fit has been couched relative to ITER baseline parameters, and $B_{\text {pen }}$ is the $q=2$ resonant component of RMS flux averaged field at the boundary, as described in section 1.1.
Thus a field threshold of $1.4 \times 10^{-4}$ in DIII-D $\beta_{\mathrm{N}}=1.8$ torque-free plasmas in figure 4 extrapolates to $1.7 \times 10^{-4}$ in ITER baseline H-modes. This is significantly lower even than the thresholds expected for ITER's low density ohmic regime-projections for ITER-FDR were $1.25 \times 10^{-4}$ in terms of vacuum $2 / 1$ field [3], but rose to $1.8 \times 10^{-4}$ for the final ITER design, equivalent to $2.8 \times 10^{-4}$ in the measure used here$50 \%$ higher than the threshold in torque-free $\mathrm{H}$-modes. Further work is needed to confirm density scalings, and test at the lower $q_{95}$ of ITER. However, if these do not yield a significant (and somewhat unexpected) improvement to counteract the low absolute levels observed in DIII-D, then this suggests a pressing need to re-evaluate whether ITER's error field correction system can meet the challenge, particularly as it was designed to minimize $m=1-3$ perturbations, whereas the current understanding [6] is that higher $m$ source perturbations are most important in driving both the $2 / 1$ response at $q=2$, and other responses across the plasma, due to the way in which the field couples to the plasma by driving a stable ideal kink instability.

\section{Conclusions and implications for future devices}

New effects have been identified in the interaction of $3 \mathrm{D}$ fields with tokamak plasmas, with the response to such fields enhanced at lower rotation or in proximity to the tearing mode $\beta_{\mathrm{N}}$ limit. This leads to regimes previously considered robust to the effects of such fields, such as the ITER baseline, becoming highly sensitive to them. Observations suggest this interaction occurs through an increased resistive response due to a breakdown of plasma screening. Modelling with the MARS-F code confirms the interpretation with the usual plasma screening response breaking down in low rotation plasmas and a tearing response developing, opening the door to additional sensitivities to $\beta$ and the current profile.

This enhanced response to $3 \mathrm{D}$ fields enables new mechanisms of mode formation, such as through magnetic braking changing underlying tearing stability, as well as potentially enhancing the conventional error field penetration process. The criterion for mode formation appears related to the degree of braking induced in the plasma. When close to the tearing mode $\beta$ limit, decreased rotation shear can destabilize the rotating mode, while at lower $\beta$, higher levels of field are needed to stop rotation and drive locked modes directly.

The nature of the interaction of the various fields applied depends on the rotation regime and field structure. At high rotation, $n=1$ fields that contain significant pitch resonant components with the plasma lead to levels of braking and lowering of tearing mode $\beta$ limits that are similar to effects from dominantly non-resonant $(n=3)$ fields. This suggests an interaction principally of the non-resonant components in both cases, through the effects of neoclassical toroidal viscosity, while any resonant parts of the field remain shielded out. Conversely, the resistive response observed at low rotation necessarily arises from components of the field that are locally pitch resonant with the core plasma. Thus, while the $\beta$ related amplification and braking effects observed at high rotation might be through the driving of kink modes (the 'ideal' plasma 
response, with generally lower field pitch than the core plasma) and lead to braking torques across the plasma, the response at low rotation will also be associated with a breakdown in shielding, and sensitivities in tearing mode stability, and lead to localized braking at rational surfaces. This suggests that minimizing pitch resonant components in the error field, in addition to kink resonant components, may be important when considering low rotation plasmas.

Based on these sensitivities, new scalings have been obtained for the tolerable level of field in ITER-baseline-like torque-free plasmas at $\beta_{\mathrm{N}}=1.8$, though with elevated $q_{95}$ (4.4 cf 3.1 in ITER). These show dependences similar to ohmic regimes, but with a much lower absolute value, and with an additional linear dependence on proximity to the tearing $\beta_{\mathrm{N}}$ limit (which itself depends on rotation). Fields have been expressed in terms of the dominant eigenmode at the plasma boundary that resonates with the $q=2$ surface, including ideal plasma response, providing a basis for evaluating thresholds from any form of resonant error field, by calculating its overlap integral at the plasma boundary with the dominant eigenmode for driving a $2 / 1$ response at $q=2$ [11].

The results highlight the importance of now carefully re-evaluating error field correction capability for ITER. In particular, it is important to assess the total expected error field in the correct physics variables. It is also vital to start to assemble a deeper understanding of error field correction and side-band roles, as corrections achieved to date on other experiments are less successful (for example in reducing density limits), possibly by an order of magnitude, than ideal response models predict. These aspects are critical to understanding both the degree of correction required, and whether this needs one, two or more arrays of correction coils. The results also have wider ramifications for tokamaks, indicating a potential for increased 3D field sensitivity when plasmas are close to tearing instability, especially with low injected torque, but also the possibility to raise thresholds through increases in rotation or improving underlying tearing stability, for example by tuning the current profile or applying torque.

\section{Acknowledgment}

This work was supported in part by the US Department of Energy under DE-FC02-04ER54698, DE-AC02-09CH11466, DE-FG02-04ER54461 and DE-FG02-07ER54917.

\section{References}

[1] Fitzpatrick R. et al 1991 Phys. Fluids B 644

[1b] Fitzpatrick R. et al 1993 Nucl. Fusion 331049

[2] Buttery R.J. et al 2000 Nucl. Fusion 40807

[3] Buttery R.J. et al 1999 Nucl. Fusion 391827

[4] Boozer A.H. 2001 Phys. Rev. Lett. 865059

[5] Garofalo A.M. et al 2002 Phys. Plasmas 91997

[6] Park J.-K. et al 2007 Phys. Rev. Lett. 99195003

[7] Reimerdes H. et al 2009 Nucl. Fusion 49115001

[8] La Haye R.J. et al 1992 Nucl. Fusion 322119

[9] Buttery R.J. et al 2008 Phys. Plasmas 15056115

[10] La Haye R.J. et al 2010 Phys. Plasmas 17056110

[11] Park J.-K. et al 2010 Proc. 23rd Fusion Energy Conf. (Daejeon, Korea, 2010) (Vienna: IAEA) EXS/P5-12

[12] Scoville J.T. et al 2003 Nucl. Fusion 43250

[13] Jackson G.L. et al 2003 Proc. 30th EPS Conf. on Plasma Physics and Controlled Fusion (St Petersburg, Russia, 2003) vol 27A (Geneva: European Physical Society) p 4.47

[14] Lanctot M.J. et al 2011 Measurement and modeling of three-dimensional equilibria in DIII-D Phys. Plasmas at press

[15] Reimerdes H. 2010 Proc. 23rd Fusion Energy Conf. (Daejeon, Korea, 2010) (Vienna: IAEA) EXS/5-4

[16] Brennan D.P. et al 2002 Phys. Plasmas 92998

[17] Bondeson A., Vlad G. and Lütjens H. 1992 Fluids B 41889

[18] Liu Y.Q. et al 2000 Phys. Plasmas 73681

[19] Hegna C.C., Callen J.D. and La Haye R.J. 1999 Phys. Plasmas 6130

[20] Liu Y.Q., Kirk A. and Nardon E. 2010 Phys. Plasmas 17122502

[21] Li D. 1998 Phys. Plasmas 51231

[22] Fitzpatrick R. 1998 Phys. Plasmas 53325

[23] Gerhardt S.P. et al 2009 Nucl. Fusion 49032003

[24] Sontag A.C. et al 2007 Nucl. Fusion 471005

[25] Sauter O., Angioni C. and Lin-Liu Y.R. 1999 Phys. Plasmas 62834

[26] Sauter O., Angioni C. and Lin-Liu Y.R. 2002 Phys. Plasmas 95140 